\title{
Multi-Objective Optimization of a Spring Diaphragm Clutch on an Automobile Based on the Non-Dominated Sorting Genetic Algorithm (NSGA-II)
}

\author{
Junchao Zhou ${ }^{1,2, *}$, Chun Wang ${ }^{1,2}$ and Junjun Zhu ${ }^{3}$ \\ 1 School of Mechanical Engineering, Sichuan University of Science and Engineering, Zigong 643000, China \\ 2 Sichuan Provincial Key Lab of Process Equipment and Control Engineering, Zigong 643000, China; \\ susewangchun@163.com \\ 3 School of Mechanical and Vehicular Engineering, Beijing Institute of Technology, Beijing 100081, China; \\ junjun_zhu_88@163.com \\ * Correspondence: zhou1987g@suse.edu.cn
}

Academic Editor: Fazal M. Mahomed

Received: 25 September 2016; Accepted: 17 November 2016; Published: 3 December 2016

\begin{abstract}
The weight coefficients of the diaphragm spring depend on experiences in the traditional optimization. However, this method not only cannot guarantee the optimal solution but it is also not universal. Therefore, a new optimization target function is proposed. The new function takes the minimum of average compress force changing of the spring and the minimum force of the separation as total objectives. Based on the optimization function, the result of the clutch diaphragm spring in a car is analyzed by the non-dominated sorting genetic algorithm (NSGA-II) and the solution set of Pareto is obtained. The results show that the pressing force of the diaphragm spring is improved by $4.09 \%$ by the new algorithm and the steering separation force is improved by $6.55 \%$, which has better stability and steering portability. The problem of the weight coefficient in the traditional empirical design is solved. The pressing force of the optimized diaphragm spring varied slightly during the abrasion range of the friction film, and the manipulation became remarkably light.
\end{abstract}

Keywords: clutch; diaphragm spring; multi-objective; optimization; NSGA-II

\section{Introduction}

The diaphragm spring clutch is widely used in automation because of good nonlinear characteristics [1]. The diaphragm spring load-deformation characteristic curve is illustrated in Figure 1. The point $A$ represents the wear limit point, the point $B$ represents the new working point, and the point $C$ represents the separating point. $\lambda_{1 B}$ is the diaphragm spring deformation of the point $B$. However, there are some problems with the clutch, such as frequent usage, high labor intensity of operators, incomplete separation during long term operation and unstable connection with easiness of slipping [2]. Because of the above results, the operating pressure of the clutch pedal stroke and many other parameters in the design are often unreasonable. This maymean that clutch separation is not complete and the clutch slips. Crucially, the performance of the clutch diaphragm spring directly affects the above parameters and it has important practical engineering optimal design implications. 


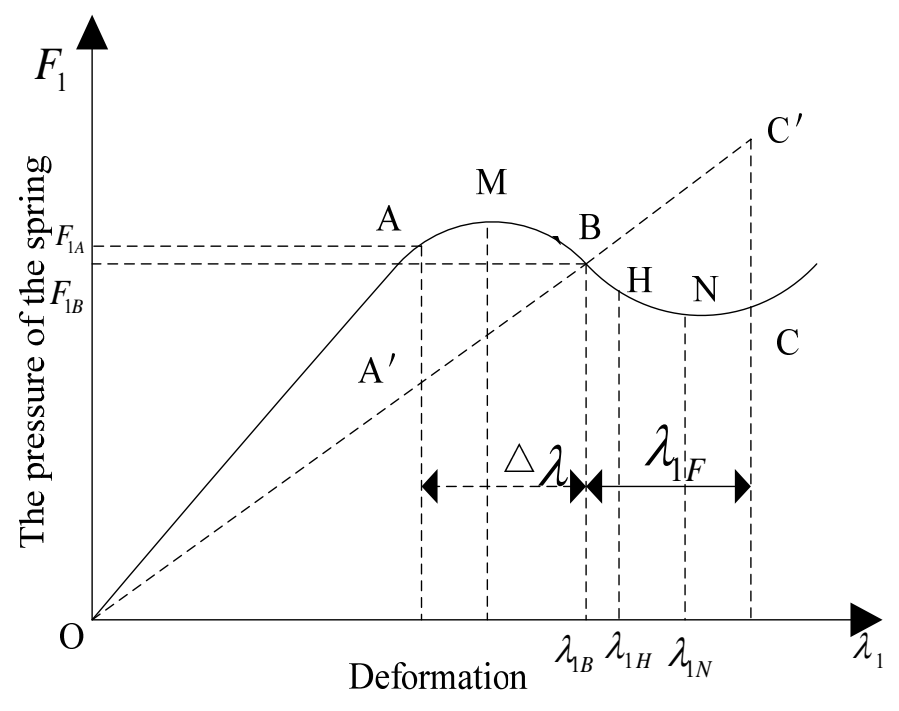

Figure 1. Characteristics of loading-deflection diaphragm spring. A: wear limit point; B: new working point; $H$ : working point; $C$ : separating point; $N$ : lowest point; $\lambda_{1 \mathrm{~B}}$ : diaphragm spring deformation of point $B ; \lambda_{1 \mathrm{H}}$ : diaphragm spring deformation of point $\mathrm{H} ; \lambda_{1 \mathrm{~N}}$ : diaphragm spring deformation of point $\mathrm{N} ; \mathrm{F}_{1 \mathrm{~A}}$ : diaphragm spring's working pressure of working point $A ; F_{1 B}$ : diaphragm spring's working pressure of working point $\mathrm{B}$.

Many scholars still show akeen interest in the diaphragm spring's optimization design and havedone much research work on it. The authors of $[3,4]$ take ideal comprehensive performance requirements of a clutch diaphragm spring as the objective function and establish a new mathematical model. The authors of $[5,6]$ present a sensitivity design method based on traditional design theory and establish an optimization model, considering the controllable variables and uncontrollable parameters variation on the influence of the diaphragm spring optimization. The method is used in the parallel optimization design of the friction type diaphragm spring clutch. The balance between each optimal target's weight coefficient is studied in $[7,8]$, the concept of parallel optimization and its application model is also proposed. The authors of [9] set up a new multi-objective optimization design, taking the minimum of average compressing force of spring within the scope of the friction slice wear and the driver's minimum manipulating force on separating bearings as optimization objectives. They also make some analysis using algorithms combined with the genetic algorithm. The optimum mathematical model of a light carrying truck's diaphragm spring clutch is established in Ref. [10]. The optimal solution set of a multi-objective optimization model of a diaphragm spring was gained using the multidisciplinary optimization software ISIGHT [11,12]. According to the above, it can be seen that many scholars have begun to study the theory of optimization design and its applications. In respect of multi-objective optimization design, multi-objective optimization is usually transformed into single optimization by each optimal target's weight coefficient. However, there is no exact method to obtain the true optimal value of each optimal target's weight coefficient and it usually depends on the scholar's experience and should be adjusted many times. This method not only cannot guarantee the optimal solution but also is not universal.

In this paper, we are concerned with the automobile clutch diaphragm spring and current related research. We aim to study the separation stroke average of the minimum separation of control force and the pressing force change minimum as two multi-objective optimization models. At the same time, the Pareto solution set of the multi-objectives is obtained under the NSGA-II. The problem of the weight coefficient in the traditional empirical design is solved. The pressing force of the optimized diaphragm spring varied slightly during the abrasion range of the friction film, and the manipulation became remarkably light. This method is verified to be correct and effective by simulations. 
This paper is organized as follows: in Section 2, we provide a theoretical analysis. Section 3 presents the NSGA and multi-objective solution. The comparison of the NSGA-II simulations are shown in Section 4. Section 5 concludes the paper with possible extensions in the future.

\section{Diaphragm Spring Multi-Objective Mathematical Model}

\subsection{Multiple Objective Functions}

The main structural parameters of a diaphragm spring are illustrated in Figure 2. The absolute value of the pressing force $\left|F_{b}-F_{a}\right|$ is the lowest force to ensure a reliable pressing force in the range of the wear limit. At the same time, the average value of the separation force of bearings in the separation process should be as small as possible to ensure light manipulation. Therefore, the absolute value of the pressing force change to separate the spring is selected as the first optimization target $F_{1}(X)$ to transfer torque reliably. The minimum average driven disc spring-loaded force is chosen as the second optimization target $F_{2}(X)$. Therefore, the objective function can be expressed as [8]:

$$
F=\left\{\begin{array}{l}
F_{1}(X)=\left|F_{b}-F_{a}\right| \\
F_{2}(X)=\min \frac{1}{N} \sum_{i=1}^{n}\left(F_{b i}-F_{b}\right)
\end{array}\right.
$$

where $F_{b}$ is the diaphragm spring's working pressure of working point $\mathrm{B} ; F_{a}$ is the working pressure of diaphragm spring when the wear reaches the limit point, and $F_{b i}$ is the pressing force corresponding to point $N$.
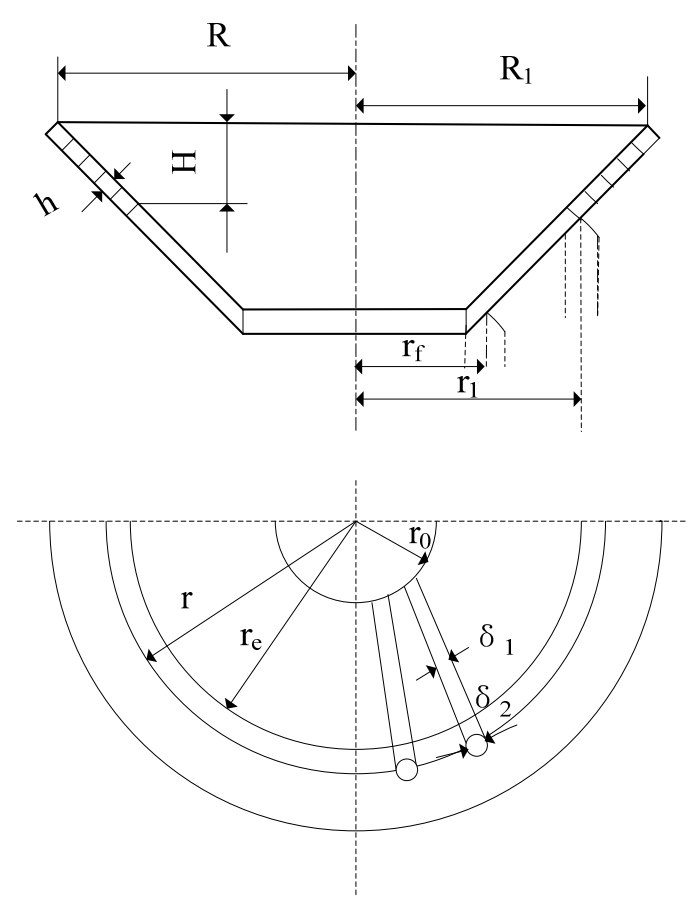

Figure 2. Main structural parameters of diaphragm spring. $H$ : cone height of the disc spring; $h$ : spring diaphragm thickness; $R_{1}$ : radius of the pressure plate's loading point; $r_{1}$ : radius of loading point of the support ring; $r_{0}$ : diameter of spring diaphragm's small end; $r_{f}$ : action radius of the separating bearing force; $\delta_{1}$ : width of the small end; $\delta_{2}$ : window width; $r_{e}$ : radius of the small end; $R$ : big end radius of the disc spring; $r$ : small end radius of the disc spring.

\subsection{Design Variables}

Diaphragm spring design is complex. For the formula of the loading-deflection diaphragm spring, six variables are needed [9]. The lateral position of the new point $B$ and corresponding 
amount of deformation $\lambda_{1 B}$ has a greater impact on the entire characteristic curve. So the main design parameters are seven parameters according to the objective function [8], namely, $h, R, r, R_{1}, r_{1}$ and $\lambda_{1 B}$. The diaphragm spring design variables matrix $X$ is determined as:

$$
X=\left[x_{1}, x_{2}, x_{3}, x_{4}, x_{5}, x_{6}, x_{7}\right]^{T}=\left[H, h, R, r, R_{1}, r_{1}, \lambda_{1 B}\right]^{T}
$$

\subsection{Constraints}

(1) The new pressing force of the spring $F_{a}$ should be equal to the required clamping force $F_{Y}$ [3]:

$$
F_{a}=F_{Y}
$$

(2) To ensure the stable operation after the friction plate, the pressing force of the spring work after the damaged $F_{1 A}$ should not be less than the new corresponding parameters $F_{1 B}$ [3]:

$$
F_{1 A} \geq F_{1 B}
$$

(3) Considering the depth-thickness ratio $H / h$ has impactions on the load-deformation curve of the diaphragm, it should be met within a certain range. At the same time, the diaphragm spring initial cone angle $\alpha_{0}$ should be controlled within a certain range [8]. That is:

$$
\left\{\begin{array}{l}
1.7 \leq H / h \leq 2.2 \\
9^{\circ} \leq \alpha_{0} \leq 15^{\circ}
\end{array}\right.
$$

(4) For the friction plate on the pressing force distribution, the radius of the big end of the friction plate $R_{1}$ should be taken between the mean radius and the outside diameter of the friction plate, according to engineering experience [9]:

$$
\left\{\begin{array}{l}
\text { pull: }(D+d) / 4 \leq r_{1} \leq D / 2-3 \\
\text { push: }(D+d) / 4 \leq R_{1} \leq D / 2-3
\end{array}\right.
$$

(5) In order to meet the structural arrangement of the diaphragm spring actual situation, the big end of the radius $R$, the support ring radius $R_{1}$, load radius $r_{1}$ and inner diameter $r$ should be in a certain range [9]:

$$
\left\{\begin{array}{l}
1 \leq R-R_{1} \leq 7 \\
0 \leq r_{1}-r \leq 6 \\
0 \leq r_{1}-r_{0} \leq 6
\end{array}\right.
$$

(6) To ensure the working point, the wear point and separation point should be distributed relatively reasonabley the new location $\lambda_{1 B}$ should meet the following conditions [8]:

$$
0.8 \leq \frac{\lambda_{1 B}}{H}\left(\frac{R-r}{R_{1}-r_{1}}\right) \leq 1.0
$$

(7) The work pressing force of the new designed diaphragm $F_{B}$ should be not less than the force $F_{C}$ in the separation process [9]:

$$
F_{B} \geq F_{C}
$$

(8) In order to make diaphragm spring satisfy a certain leverage ratio during the separation, the ratio of the outer diameter to inner diameter should be met [3]:

$$
\left\{\begin{array}{l}
1.2 \leq R / r \leq 1.3 \\
3.0 \leq\left(r_{1}-r_{f}\right) /\left(R_{1}-r_{1}\right) \leq 4.0
\end{array}\right.
$$


(9) To take advantage of spring material, part size should meet certain requirements, according to engineering experience [8]:

$$
\left\{\begin{array}{l}
1.2 \leq R / r \leq 1.35 \\
70 \leq 2 R / h \leq 100 \\
3.5 \leq R / r_{0} \leq 5.0
\end{array}\right.
$$

(10) The highest point of the tensile stress $\sigma_{A \max }\left(\sigma_{C \max }\right)$ in the bottom of the $A$ (or $C$ ) dangerous parts of the diaphragm spring separating finger holes should meet a strength condition [9]:

$$
\left\{\begin{array}{l}
\sigma_{A \max } \leq\left[\sigma_{A}\right] \\
\sigma_{C \max } \leq\left[\sigma_{C}\right]
\end{array}\right.
$$

(11) During the diaphragm spring manufacturing process, there are some major dimensions machining errors, the error during assembly process should meet certain requirements [9]:

$$
\left(\Delta F_{h}+\Delta F_{t}+\Delta F_{R}+\Delta F_{r}\right) / F_{b} \leq 0.05
$$

\section{NSGA-II Algorithm and Multi-Objective Solution}

In 2002, Deb et al. proposed the NSGA-II, and introduced elite strategies to improve the efficiency of the algorithm searches and robustness $[13,14]$.

In practical engineering, multiple indicators may be involved in many optimization problems $[15,16]$, e.g., the diaphragm spring requires that not only the absolute value of the pressing force $\left|F_{b}-F_{a}\right|$ is the lowest value that ensures a reliable pressing force in the range of the wear limit but, in addition, the average value of the separation force of bearings in the separation process should be as small as possible to ensure light manipulation. These two or more design specifications achieve an optimal value that is termed multi-objective optimization. In the diaphragm spring multi-objective optimization problem, each target cannot achieve the optimal simultaneously. In the conventional processing multi-objective optimization problem, the normalization method is usually used, such as the weighting coefficient method and the hierarchical sequence method, by building an evaluation function and eventually transforming the multi-objective optimization problem into a single objective optimization solution. However, the normalization method often relies on the experience of policy makers. Since each target objection of diaphragm spring is interrelated and constrained, even a target is improved by reducing another target as the cost $[17,18]$. Many objective solutions are typically non-inferior solution sets. Therefore, it is important to search for the Pareto set in the multi-objective optimization of the diaphragm spring. Figure 3 shows the flow of the NSGA-II algorithm. The main steps of NSGA-II algorithm used in this paper are as follows: (Initial population) create a random population of $\mathrm{N}$ chromosome in the population. (Calculate the objective function) calculate the multi-objective function of spring diaphragm. (Satisfied termination criteria) check whether the termination criterion is satisfied or not. If yes, the population is terminated, otherwise, adjust the fitness distribution. (Fitness distribution) evaluate the multi-objective fitness of each chromosome in the population. (Genetic operation and insert parent) generate a new population. 


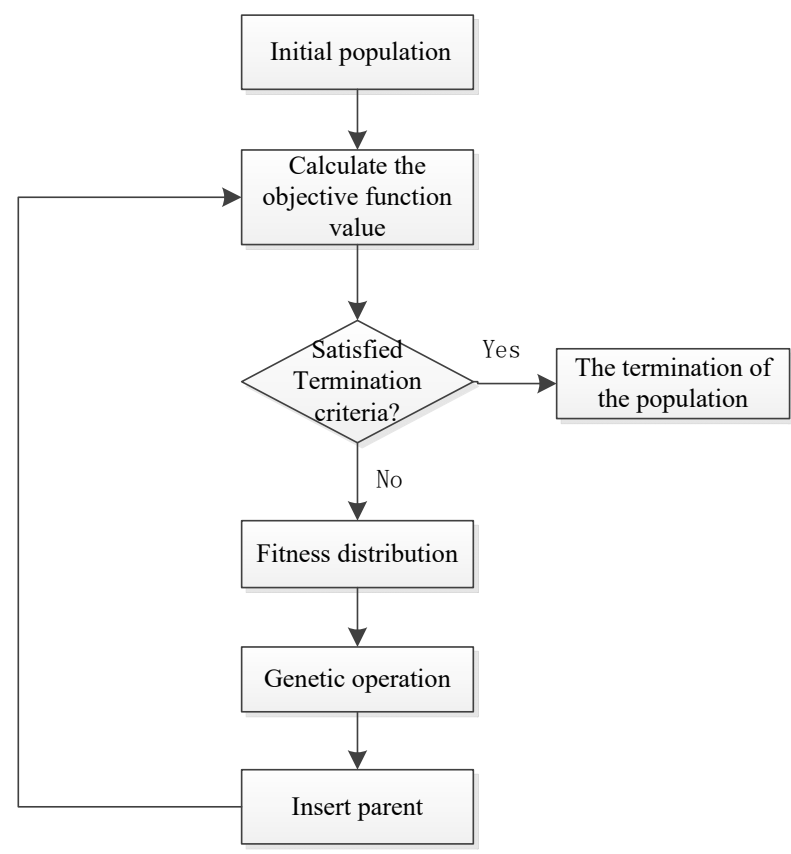

Figure 3. Non-dominated sorting genetic algorithm (NSGA)-II; algorithm optimization process.

\section{Results and Discussion}

The design of certain new type of pull diaphragm spring clutch optimization is taken as an example. The parameters are as follows: Maximum engine torque is $1150 \mathrm{~N} \cdot \mathrm{m}$, reserve coefficient $\beta$ is 1.8 , the friction factor is 0.3 , and the diaphragm spring material is 60 si2MnA. Allowable stress is 1500-1700 MPa; Poisson's ratio is 0.3 , the wear limit is $3.2 \mathrm{~mm}$ and separation stroke is $3.5 \mathrm{~mm}$. The specific target variables of the experiment are shown in Table 1. The NSGA-II algorithm parameter setting is shown Table 2 .

Table 1. Experimental parameters design of optimization variables.

\begin{tabular}{ccc}
\hline Plan & Objective Function & Algorithm \\
\hline A & $\alpha F_{1}(X)+\beta F_{2}(X)$ & Penalty function method \\
B & $\alpha F_{1}(X)+\beta F_{2}(X)$ & genetic algorithm \\
C & $F_{1}(X), F_{2}(X)$ & NSGA-II \\
\hline
\end{tabular}

Notes: $\alpha, \beta$ : weight coefficients, $\alpha+\beta=1 ; F_{1}(X)$ : target $1 ; F_{2}(X)$ : target 2 .

Table 2. NSGA-II algorithm parameter setting.

\begin{tabular}{ccccc}
\hline $\begin{array}{c}\text { Population } \\
\text { Size }\end{array}$ & $\begin{array}{c}\text { Stop } \\
\text { Algebra }\end{array}$ & $\begin{array}{c}\text { Fitness Function } \\
\text { Value Deviation }\end{array}$ & $\begin{array}{c}\text { Optimal Front End } \\
\text { Individual Coefficient }\end{array}$ & $\begin{array}{c}\text { Maximum } \\
\text { Iterative Algebra }\end{array}$ \\
\hline 100 & 200 & $1 \times 10^{-100}$ & 0.3 & 200 \\
\hline
\end{tabular}

The model is solved by using the penalty function method [8], the genetic algorithm [9] and NSGA-II. The constrained optimization problem is transformed into an unconstrained condition by the penalty function method. The genetic algorithm has very good convergences such as calculation of accuracy requirements. However, the penalty function method and the genetic algorithm are used to solve the multi-objective optimization problem into a single objective function. The weight coefficient is needed to balance the relationship between multiple objectives. The weight coefficients usually depend on experience. So, the multiple objectives are solved by the NSGA-II. The Pareto solution set by NSGA-II is shown in Figure 4. 


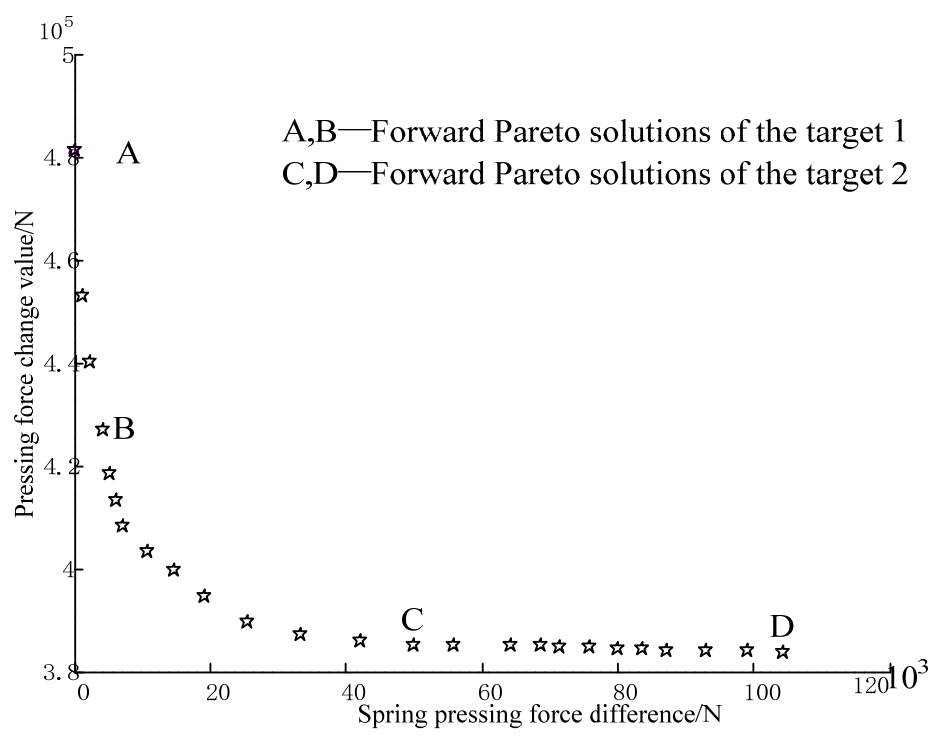

Figure 4. Multi-objective optimization Pareto optimal solution set.

From Figure 4, it is found that the Pareto frontier A and D stand for the absolute value of the difference between the minimum point and the spring pressing force change value, before and after the spring-loaded force of the diaphragm spring breaks the disc wear. During AB there are great changes in discs wear spring-loaded forces due to small changes of the spring pressing force. During CD there are great changes of the spring pressing force because of small changes in the disc wear loaded forces. Therefore, the midpoint near the BC is chosen as a result of multi-objective optimization. The optimal results of the diaphragm spring are shown in Tables 3 and 4.

Table 3. Comparison of optimized structural parameters with original ones.

\begin{tabular}{cccccccc}
\hline Plan & $\boldsymbol{H}(\mathbf{m m})$ & $\boldsymbol{h}(\mathbf{m m})$ & $\boldsymbol{R}(\mathbf{m m})$ & $\boldsymbol{r}(\mathbf{m m})$ & $\boldsymbol{R}_{\mathbf{1}}(\mathbf{m m})$ & $\boldsymbol{r}_{\mathbf{1}}(\mathbf{m m})$ & $\boldsymbol{\lambda}_{1 \boldsymbol{B}}$ \\
\hline Original & 5.8 & 2.93 & 145.7 & 116.8 & 143.66 & 116.1 & 4.80 \\
A & 5.24 & 2.80 & 140.00 & 115.00 & 138.68 & 115.00 & 4.21 \\
B & 5.20 & 2.80 & 140.04 & 115.18 & 138.80 & 114.00 & 4.02 \\
C & 5.21 & 2.81 & 140.35 & 115.48 & 140.66 & 114.50 & 4.01 \\
\hline
\end{tabular}

Notes: $H$ : cone height of the disc spring; $h$ : spring diaphragm thickness; $R$ : big end radius of the disc spring; $r$ : small end radius of the disc spring; $R_{1}$ : radius of the pressure plate's loading point; $r_{1}$ : radius of loading point of the support ring; $\lambda_{1 B}$ : new deformation of point $B$.

Table 4. Comparison of optimized performance parameters with original ones.

\begin{tabular}{cccccc}
\hline Plan & $\boldsymbol{F}_{\boldsymbol{b}} \mathbf{( N )}$ & $\boldsymbol{F}_{\boldsymbol{a}}(\mathbf{N})$ & $\boldsymbol{F}_{\boldsymbol{c}}(\mathbf{N})$ & $\left|\boldsymbol{F}_{\boldsymbol{b}}-\boldsymbol{F}_{\boldsymbol{a}}\right| \mathbf{( N )}$ & $\left|\boldsymbol{F}_{\boldsymbol{b}}-\boldsymbol{F}_{\boldsymbol{a}}\right| /\left|\boldsymbol{F}_{\boldsymbol{b}}\right| \mathbf{( \% )}$ \\
\hline Original & 5226 & 5925 & 3817 & 699 & 13.37 \\
A & 4834 & 5185 & 3709 & 351 & 7.23 \\
B & 4757 & 5016 & 3715 & 259 & 5.44 \\
C & 4422 & 4603 & 3567 & 181 & 4.09 \\
\hline
\end{tabular}

Notes: $F_{b}$ : working pressure of working point $\mathrm{B} ; F_{a}$ : working pressure of diaphragm spring when the wear reaches the limit point; $F_{c}$ : separation force; $\left|F_{b}-F_{a}\right| /\left|F_{b}\right|$ : relative rate of change of the steering separation force.

The optimal results of diaphragm spring are illustrated in Tables 3 and 4. From Tables 3 and 4 it is found that the smaller the deformation of the big-deformation $\lambda_{1}$, the longer the lifespan of the clutch. So the lifespan of the diaphragm spring by the NSGA-II is better than the penalty function method and the genetic algorithm. The wear range $\left|F_{b}-F_{a}\right|=181 \mathrm{~N}$ and relative rate of change $\left|F_{b}-F_{a}\right| /\left|F_{b}\right|=4.09 \%$, which is far less than the 10\% [9], improving the compaction force stability. 
In addition, the steering separation force of the clutch after optimization is the smallest. The relative rate of change of the steering separation force is $|3817-3567| / 3817=6.55 \%$, so the operating flexibility is improved.

The final optimal results of the load deformation characteristic curve based on the Table 2 are illustrated in Figure 5. From Figure 5, it is found that the friction plate abrasion clamping force of the diaphragm spring within the limit range in the scheme A is greatly reduced, but the separation of driver control force average change is increased slightly. So, the optimization of the scheme A is not ideal. Compared with the original scheme, the friction plate abrasion clamping force of the diaphragm spring within the limit range in scheme B is greatly reduced too. In addition, separation of the driver control force average also decreased, so the effect is good. Compared to the scheme $C$ with the original scheme, the friction plate abrasion clamping force of the diaphragm spring within the limit range is drastically reduced, and separation of the driver control force is greatly reduced. The separation performance improved significantly compared with the first two schemes. Comparing A, B and C, the three schemes improve a little, but clamping force in scheme $C$ is more gentle. At the same time, the pedal force that needs to be overcome to separate the drives control force is smaller, so separation is more portable and the comprehensive performance of the last program is the most comprehensive and comparative one of the three integrated programs.

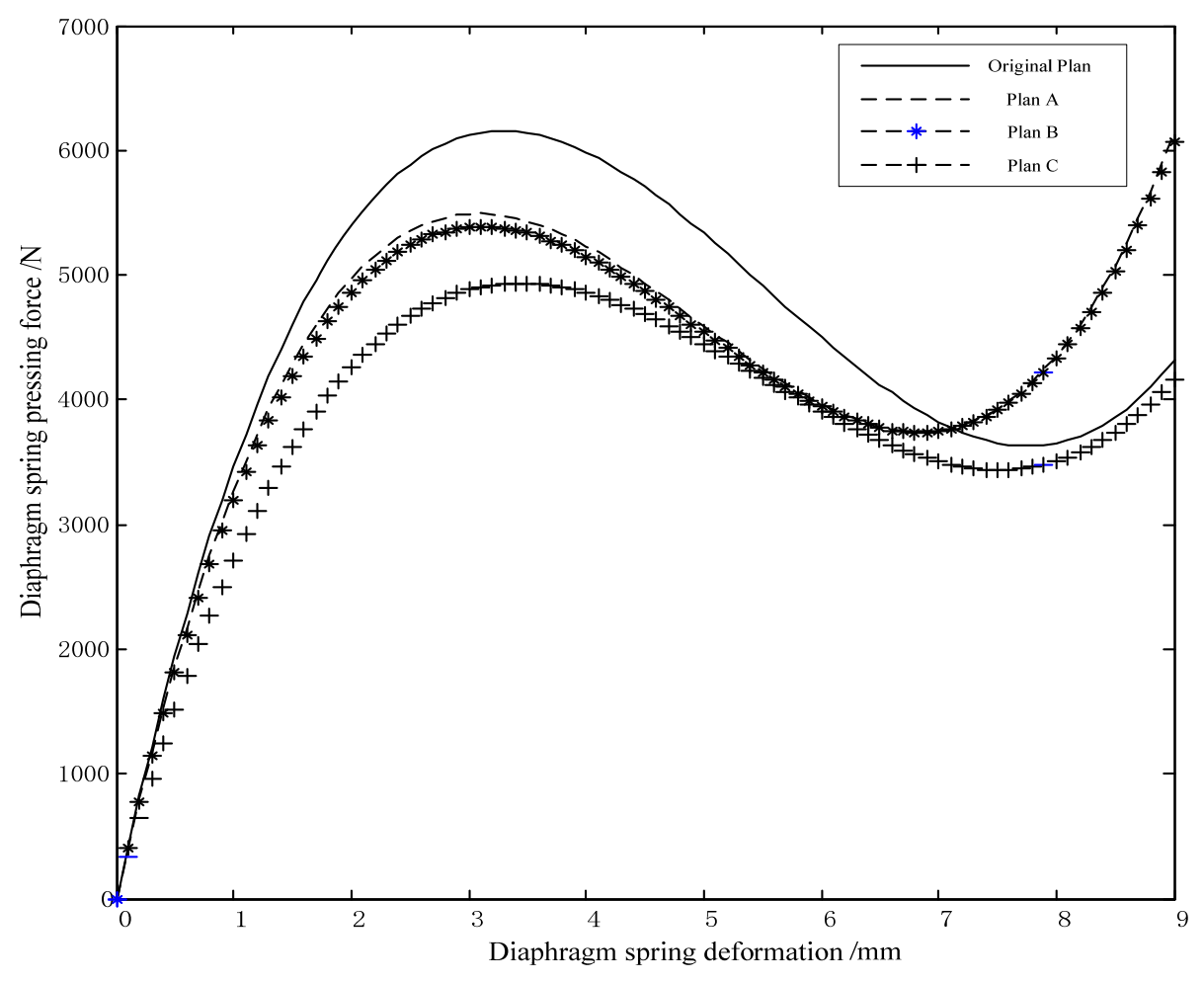

Figure 5. Characteristic curve of the diaphragm spring.

From Tables 3 and 4, and Figure 5, in can be seen that the pressing force of the optimized diaphragm spring varied slightly during the abrasion range of the friction film, and the manipulation became remarkably light. The problem of the weight coefficient in the traditional empirical design is solved. The new optimization model is proved correct and effective, and the purpose of optimization is achieved.

\section{Conclusions}

In this paper, the multi-objective optimizations of a clutch diaphragm spring are carried out based on NSGA-II. A new optimization target function is proposed. The new function takes the minimum 
average change in compress force of the spring and the minimum force of the separation as total objectives. The results show that the pressing force of the diaphragm spring by the new algorithm is improved by $4.09 \%$ and the steering separation force is improved by $6.55 \%$, which has better stability and steering portability. The results show that the optimal purpose can be achieved in the given case. Comparing the NSGA-II with the penalty function method and the genetic algorithm indicates that the multi-objective can lead to a more desirable design than the single-objective optimization. Future work may include further development of NSGA-II by adding other constraints, e.g., reliability, or other objective functions, e.g., weight; use of these algorithms on other clutch optimization problems may also be valuable.

Acknowledgments: This research is supported by the research project of the Sichuan Provincial Key Lab of Process Equipment and Control Engineering (GK201508).

Author Contributions: Chun Wang analyzed the data; Junjun Zhu contributed analysis tools; Junchao Zhou wrote the paper.

Conflicts of Interest: The authors declare no conflict of interest.

\section{References}

1. Xia, C.; Zhu, P.; Zhang, M.; Gao, X.; Lu, L. Dispersion of the mechanical parts performance indicators based on the concept of random vector. Chin. J. Mech. Eng. 2012, 25, 153-159. [CrossRef]

2. Haugen, E.B. Probabilistic Mechanical Design; John Wiley \& Sons: New York, NY, USA, 1980.

3. Wu, Y.-H. Multi-objective optimization design of vehicle clutch diaphragm spring. In Proceedings of the International Conference on Intelligent Computation Technology and Automation, Changsha, China, 10-11 October 2009; pp. 193-197.

4. Tripathi, K. Some Design-Objectives and Design-Guidelines for Automotive Friction Clutch Based on Clutch Engagement Dynamics. J. Inst. Eng. 2014, 95, 51-61. [CrossRef]

5. Amisano, F.; Ercole, G.; Mattiazzo, G.; Mauro, S.; Serra, G.; Velardocchia, M. The influence of the push plate mechanical characteristic on torque transmissibility in diaphragm spring. In Proceedings of the 3rd International AIMETA Tribology Conference, Vietri sul Mar, Italy, 18-20 September 2002; Volume 24, pp. 98-108.

6. Xi, J.-Q.; Lu, J.; Pan, C. Modeling and simulation of the static characteristics of diaphragm spring clutches. J. Beijing Inst. Technol. 2015, 24, 188-193.

7. Kaya, N. Optimal design of an automotive diaphragm spring with high fatigue resistance. Int. J. Veh. Des. 2006, 40, 126-143. [CrossRef]

8. Zhao, L.-J.; Liu, T.; Song, B.-Y. Optimum design of automobile diaphragm spring clutch. In Proceedings of the IEEE Vehicle Power and Propulsion Conference, Harbin, China, 3-5 September 2008; pp. 1421-1427.

9. Ran, Z.Y.; Zhao, S.E.; Yu, L.I. Optimum Design for the Diaphragm Spring Clutch on Automobile with Genetic Algorithms. J. Chongqing Univ. 2003, 26, 77-81.

10. Mohamad, N.; Iovenitti, P.; Vinay, T. Modelling and Optimisation of a Spring-Supported Diaphragm Capacitive MEMS Microphone. Engineering 2010, 2, 762-770. [CrossRef]

11. Ye, Z.; Kaiyuan, Y. A Study of Belleville Spring and Diaphragm Spring in Engineering. J. Appl. Mech. 1990, 57, 1026-1031.

12. Chan, C.-K.; Lai, W.-C.; Wu, M.; Wang, M.-Y.; Fang, W. Design and Implementation of a Capacitive-Type Microphone with Rigid Diaphragm and Flexible Spring Using the Two Poly Silicon Micromachining Processes. IEEE Sens. J. 2011, 11, 2365-2371. [CrossRef]

13. Pasandideh, S.H.R.; Niaki, S.T.A.; Asadi, K. Bi-objective optimization of a multiproduct multi-period three-echelon supply chain problem under uncertain environments NSGA-II and NRGA. Inf. Sci. 2015, 292, 57-74. [CrossRef]

14. Brownlee, A.E.I.; Wright, J.A. Constrained, mixed-integer and multi-objective optimization of building designs by NSGA-II with fitness approximation. Appl. Soft Comput. 2015, 35, 114-126. [CrossRef]

15. Li, Y.; Liao, S.; Liu, G. Thermo-economic muti-objective optimization for a solar-dish Brayton system using NSGA-II and decision making. Electr. Power Energy Syst. 2015, 64, 167-175. [CrossRef] 
16. Bandyopadhyay, S.; Bhattacharya, R. Solving multi-objective parallel machine scheduling problem by modified NSGA-II. Appl. Math. Model. 2013, 37, 6718-6729. [CrossRef]

17. Nourbakhsh, A.; Safikhaniand, H.; Derakhshan, S. The comparison of multi-objective particle swarm optimization and NSGA II algorithm: Applications in centrifugal pumps. Eng. Optim. 2011, 43, 1096-1113. [CrossRef]

18. Murugan, P.; Kannan, S.; Baskar, S. Application of NSGA-II Algorithm to Single-Objective Transmission Constrained Generation Expansion Planning. IEEE Trans. Power Syst. 2009, 24, 1790-1797. [CrossRef]

(C) 2016 by the authors; licensee MDPI, Basel, Switzerland. This article is an open access article distributed under the terms and conditions of the Creative Commons Attribution (CC-BY) license (http:/ / creativecommons.org/licenses/by/4.0/). 